\title{
La thérapie cellulaire du diabète
}

\section{Le point sur les actualités}

> La possibilité de remplacer des cellules $\beta$ déficientes par de nouvelles cellules insulinosécrétantes chez le patient diabétique est démontrée par le succès de la transplantation d'îlots pancréatiques. Le manque de donneurs cadavériques a stimulé la recherche de sources alternatives de cellules $\beta$ dont le palmarès est dominé par les cellules souches pluripotentes humaines pouvant acquérir des caractéristiques quasiment identiques à celles des cellules $\beta$ et rétablir l'équilibre glycémique de souris diabétiques. Néanmoins, elles comportent un risque carcinogénique actuellement inévitable. Le pancréas adulte héberge des compartiments cellulaires aux capacités de transdifférenciation établies pouvant être exploitées pharmacologiquement in situ ou via des phases d'expansion en culture. Cette revue s'intéresse aux nouveaux développements concernant la thérapie cellulaire du diabète. <

L'Organisation mondiale de la santé (OMS) estime dans le monde à 350 millions le nombre d'individus atteints de diabète, entité nosologique qui rassemble des troubles de l'homéostasie glucidique grevés d'un risque à long terme de complications microet macrovasculaires [59] $(\rightarrow)$.

$(\rightarrow)$ Voir le Faits et Chiffres de $C$. Franc, $m / s n^{\circ} 8-9$, aoûtseptembre 2013, page 711

Parmi ceux-ci prédomine le diabète de type 2 (DT2) qui se développe sur un terrain d'insulinorésistance largement déterminé génétiquement et entraînant, à terme, une dysfonction des cellules $\beta$ insulinosécrétantes ${ }^{1}$. Dans le diabète de type 1 (DTl), qui représente $10 \%$ des cas recensés de diabète, une destruction auto-immune des cellules $\beta$ est responsable d'une progression plus rapide de l'hyperglycémie et d'un âge d'apparition plus précoce (50\% des patients sont diagnostiqués avant l'âge de 15 ans). Alors que les modifications du style de

${ }^{1}$ Les cellules $\beta$ du pancréas sont les cellules qui produisent l'insuline.

vie permettent de prévenir $60 \%$ des DT2, il n'y a, à ce jour, aucune straté-
${ }^{1}$ Laboratoire de pédiatrie, institut de recherche expérimentale et clinique, université catholique de Louvain, 1000 Bruxelles, Belgique;

${ }^{2}$ Unité d'endocrinologie pédiatrique, cliniques universitaires Saint Luc, université catholique de Louvain, 1000 Bruxelles, Belgique.

philippe.lysy@uclouvain.be

gie de prévention du DTl et les traitements actuels ne permettent pas d'atteindre les objectifs thérapeutiques tels que révélés par le dosage de l'hémoglobine glyquée (ou glycatée) $\left(\mathrm{HbAl}_{\mathrm{C}}\right)^{2}[1]$.

Le DTl est donc la cible des stratégies développées pour le remplacement des cellules $\beta$ qui ont vu le jour en 1966 à Minneapolis avec la première greffe de pancréas humain combinée à une greffe de rein [2]. Après plus de 47000 greffes d'organe (pancréas associé au rein ou pancréas seul), la technique a été nettement raffinée. Elle permet actuellement une survie des greffons d'environ $85 \%$ à 1 an post-chirurgie [3], ce qui en fait une option thérapeutique pour des patients diabétiques en insuffisance rénale [4]. Cette technique n'est malgré tout pas généralisable étant donnés les degrés de morbidité et de mortalité associés au geste chirurgical (75\% de survie à 10 ans), la nécessité d'une immunosuppression à vie, et le manque de donneurs d'organe. Ces difficultés ont stimulé la recherche pour une thérapie cellulaire du diabète fondée sur des sources cellulaires variées comme les îlots pancréatiques ${ }^{3}$, les cellules souches pluripotentes ou les cellules somatiques adultes du pancréas.

\section{Transplantation des îlots pancréatiques}

La transplantation des îlots de Langerhans du pancréas humain s'est développée dans les années 1970, comme une alternative moins invasive à la greffe de pancréas entier. Cette technique a bénéficié de la

${ }^{2}$ L'hémoglobine glyquée est une forme d'hémoglobine ayant subi une glycation, c'est-à-dire une addition non enzymatique de sucre sur la protéine. C'est un marqueur de la glycémie.

${ }^{3}$ Les îlots pancréatiques, ou îlots de Langerhans sont formés de cellules endocrines capables de synthétiser l'insuline, le glucagon, la somatostatine et le polypeptide pancréatique. 
découverte des chambres de Ricordi ${ }^{4}$ pour la purification des îlots, et du protocole de transplantation d'Edmonton (protocole établi à l'université d'Alberta, Canada) qui associe, entre autres, l'infusion des cellules isolées dans la veine porte (les îlots s'implantant alors dans le parenchyme hépatique) et l'utilisation d'un cocktail d'immunosuppresseurs incluant le tacrolimus, le sirolimus et le daclizumab. Ce dernier a fait l'objet d'une étude qui a permis à l'équipe de Shapiro de démontrer en 2000 l'efficacité de la thérapie cellulaire du diabète [5]. La technique de greffe d'îlots a subit ensuite quelques améliorations qui ont permis d'accroître la proportion de patients devenus insulinoindépendants, à $44 \%$ après 3 ans post-greffe, la majorité des patients (60\%) conservant une $\mathrm{HbAl}_{\mathrm{c}}$ en dessous de 6,5\% (soit un taux inférieur à celui caractéristique d'un diabète) [6].

Le consortium de transplantation d'îlots (CIT) a établi un registre qui permet de documenter les conditions et l'évolution des greffes cliniques: selon une étude rétrospective récente [7], la plupart des patients reçoivent actuellement un régime immunosuppresseur qui associe des anticorps anti-lymphocytes T et des inhibiteurs du TNF $\alpha$ (tumor necrosis factor $\alpha$ ) pour l'induction, et des inhibiteurs de la calcineurine et de mTOR (mammalian target of rapamycin) pour la maintenance, en post-greffe. Ces nouveaux protocoles pourraient avoir un impact important sur la survie à long terme des greffons comme en témoigne l'étude de suivi des patients EXIIST (extended immunosuppression in islet transplantation - ITN040CT) [8] qui montre, chez sept patients traités au long cours (avec le tacrolimus associé au sirolimus ou mycofenolate mofetil), la persistance d'une fonction des îlots jusqu'à 10,9 ans post-greffe, avec un impact positif sur le contrôle glycémique $\left(\mathrm{HbAl}_{\mathrm{C}}\right.$ moyenne $\left.=6,3 \%\right)$. Globalement, ces patients sont restés insulino-indépendants pendant 54 mois après transplantation sans rencontrer d'effets secondaires majeurs. Une étude actuelle du CIT (CITO7), combinant le sirolimus, les globulines anti-thymocytes et l'étanercept pour l'induction, et le tacrolimus et le sirolimus pour l'entretien, a comme objectif primaire le contrôle glycémique des patients (révélé par une $\mathrm{HbAl}_{c}$ inférieure à $7 \%$ ) et l'éviction des hypoglycémies sévères à $l$ an, après greffe d'îlots [9]. D’autres protocoles sont en cours d'évaluation à l'université de Miami, notamment l'association du granulocyte-colony stimulating factor (G-CSF) et de l'exénatide, qui, comme supplément aux protocoles classiques, semble permettre la fonction des greffons pendant une période prolongée de 4500 jours [10].

Malgré ces efforts, la transplantation d'îlots reste limitée par de nombreux obstacles avec, entre autres, le manque de donneurs cadavériques, le besoin de deux à trois pancréas nécessaires afin de collecter une masse critique d'îlots pour la transplantation, l'immunosuppression des patients induite à long terme, et la survie limitée des greffons. Certaines études évaluent actuellement la possibilité d'améliorer l'oxygénation des îlots au niveau du site de transplantation. L'équipe de Shapiro a ainsi développé un système implantable en sous-cutané

${ }^{4}$ Pour que l'enzyme utilisée pour isoler les îlots de Langerhans fonctionne, on place le pancréas dans une chambre de digestion à température contrôlée. L'organe se dissout sous l'action de l'enzyme et d'un processus mécanique de secouage. Les îlots sont ensuite séparés du tissu pancréatique par centrifugation. (constitué de cathéters en nylon) permettant une néovascularisation préalable du site de transplantation [11]. Ces chercheurs observent un effet bénéfique de ce système sur la fonctionnalité des îlots greffés et sur leur capacité à normaliser les glycémies chez des souris diabétiques transplantées. D'autres pistes sont en cours d'analyse, comme l'utilisation du vascular endothelial growth factor (VEGF), un facteur permettant la néovascularisation [12], qui pourrait être administré localement et stimuler l'irrigation sanguine locale des îlots. Enfin, les techniques de micro- et macro-encapsulation sont en constante évolution. Elles pourraient, à terme, représenter une solution viable à l'implantation des îlots, sans recours aux protocoles d'immunosuppression [13]. Cette possibilité doit néanmoins encore faire ses preuves dans le contexte clinique.

\section{Cellules souches pluripotentes}

Les cellules souches pluripotentes (CSP ou iPS) - qu'elles soient embryonnaires ou induites - représentent le fer de lance des stratégies de production in vitro et de novo, de cellules $\beta$ fonctionnelles. Leurs avantages sont multiples: disponibilité à large échelle, efficacité de différenciation et phénotypes cellulaires établis. Après quelques hésitations en termes de conditions de culture et d'identification des cellules productrices d'insuline, le domaine des cellules souches embryonnaires (CSE ou ES) a connu un essor spectaculaire après la publication en 2006-2008 des travaux de l'équipe de ViaCyte (une compagnie spécialisée dans le développement de thérapie pour le diabète, localisée à San Diego, Californie, États-Unis) : la démonstration fut faite en effet de la capacité d'un protocole de différenciation, à plusieurs étapes (sous l'effet de petites molécules et des facteurs de croissance), d'induire la production in vitro d'une population contenant $12 \%$ de cellules sécrétant l'insuline $[14,15]$. Cependant, malgré l'augmentation de la sécrétion d'insuline par les cellules souches embryonnaires différenciées après incubation avec divers sécrétagogues, qui augmentent la sécrétion des cellules endocrines (comme le tolbutamide, le chlorure de potassium ou l'isobutylméthylxanthine), les cellules ne répondaient pas au stimulus glucosé. Le volet in vivo de ces investigations a néanmoins montré la capacité des progéniteurs pancréatiques, dérivés de CSE humaines, à continuer leur processus de différenciation durant 3 à 4 mois après transplantation dans des souris immunodéprimées. Après cette échéance, les souris traitées par la streptozotocine (qui détruit les cellules $\beta$ via le transporteur de glucose GLUT2) maintenaient une glycémie normale grâce à la sécrétion d’insuline 
humaine par les greffons de CSE différenciées - démontrant ainsi leur capacité à produire des quantités physiologiques d'insuline active. L'enthousiasme généré par ces études in vivo a été obscurci par la démonstration de la propension des CSE à former des tératomes par prolifération et différenciation incontrôlée des populations cellulaires indifférenciées [16]. Le risque repose sur la transplantation de CSE ayant accumulé, au décours de cycles de prolifération in vitro, des anomalies chromosomiques conférant un risque de cancérisation [17]. Les tentatives de sélection (par cytométrie en flux ou par biologie moléculaire) des CSE engagées dans le processus de différenciation endocrine n'ont pas permis à ce jour d'assurer l'innocuité des préparations cellulaires. La résolution des techniques de tri cellulaire n'est pas assez performante pour éliminer la faible quantité de cellules pluripotentes pourtant suffisantes pour le développement d'un phénomène tumoral.

Malgré ces difficultés, le champ d'application des CSE a été largement élargi par la publication en 2014 de deux études démontrant les capacités des CSE à acquérir des caractéristiques matures de cellules $\beta$ in vitro. L'équipe de Kieffer, à Vancouver, a ainsi publié des révisions successives du protocole développé par ViaCyte [18, 19] pour aboutir à la production, ex vivo, en 40 jours, de cellules endocrines exprimant toutes les caractéristiques de cellules $\beta$ hormis le processus calciumdépendant de sécrétion d'insuline [20]. Leurs cellules $\beta$ dérivées des CSE humaines étaient capables de guérir le diabète de souris dans un délai de deux mois, c'est-à-dire après une phase de maturation in vivo sensiblement restreinte. L'équipe de Douglas Melton a, quant à elle, obtenu des cellules $\beta$ «complètes » sur les plans phénotypique et fonctionnel, avec des rendements comparables en culture entre les cellules souches embryonnaires (CSE ou ES) et les cellules souches pluripotentes (CSP ou iPS) induites [21]. Après différenciation, ces cellules montraient des pics de sécrétion d'insuline (sous stimulus glucosé) similaires aux cellules $\beta$ primaires de pancréas adultes. Dans cette étude, l'insuline humaine est détectée dans le sérum des souris seulement deux semaines après transplantation. En outre, les greffes de cellules $\beta$ reprogrammées étaient capables de prévenir le développement spontané de l'hyperglycémie chez les souris NRG-Akita (NOD-Ragl $1^{\text {null }} \mathrm{IL}-2 \mathrm{r} \gamma^{\text {null }} \operatorname{Ins} 2^{\text {Akita }}$ ), un modèle d'hyperglycémie spontanée, qui développent normalement un diabète dans un intervalle de quatre semaines. En parallèle à ces études précliniques démontrant le potentiel des CSE pour la thérapie cellulaire du diabète, ViaCyte a commencé une étude clinique de phase 1/2a (NCT02239354) au cours de laquelle des patients diabétiques de type 1 reçoivent un implant sous-cutané constitué de progéniteurs pancréatiques (dérivés des $\mathrm{CSE}$ ) conditionnés dans un système de macroencapsulation développé par la firme (Encaptra $\left.{ }^{\circledast}\right)$. II ne fait nul doute que les résultats de cette étude clinique auront un impact au niveau international sur la recherche impliquant les CSE humaines, dont l'usage a déjà été réalisé en clinique, chez des patients atteints de dystrophie ou de dégénérescence maculaire. Une étude menée par R.P. Lanza, a permis de montrer la bonne tolérance à l'implantation sous-rétinienne de CSE humaines différenciées, chez neuf sujets [22] sans signes de prolifération incontrôlée des cellules injectées.
Malgré les succès actuels obtenus avec les CSE, leur utilisation reste grevée de difficultés encore inextricables, comme les barrières éthiques à la généralisation de l'utilisation d'embryons humains, le manque de reproductibilité des protocoles de différenciation de lignées cellulaires de CSE [23], ou l'obtention de phénotypes divergents - en l'occurrence hépatocytaires - lors de la différenciation [24]. Les CSP induites (ou iPS) ont également leurs tares, surtout au niveau coût/efficacité et modifications épigénétiques/génétiques induites par les protocoles de reprogrammation (utilisant des facteurs de transcription oncogéniques tels que c-Myc ou KIf4 [Kruppel-like factor 4]) [25]. Ces limitations, associées au risque carcinogénique, retardent l'initiation de protocoles de recherche clinique et pourraient constituer un frein à l'enrôlement de candidats volontaires à la transplantation. La valeur ajoutée des CSP induites réside dans la possibilité de produire des dérivés de cellules $\beta$ autologues, ce qui a été démontré chez des patients souffrant de DTl [26] ou de diabète monogénique (autrefois appelé «MODY» pour maturity onset diabetes of the young) $[27,60](\rightarrow)$.

$(\rightarrow)$ Voir la Synthèse de G. Vehlo et al., $m / s n^{\circ} 8-9$, aoûtseptembre 203, page 854

Dans ce dernier cas, vu la préservation de la masse des cellules $\beta$ à long terme, l'autotransplantation de CSP induites pourrait s'inscrire dans le contexte de modifications génomiques in vitro dans le but de réparer la fonction déficiente (par exemple la mutation du gène $H N F l \alpha$ [hepatocyte nuclear factor $1 \alpha$ ] qui est responsable du «MODY 3 »). Cependant, l'efficacité d'une telle technique et le rapport coûts/bénéfices pour le patient nécessitent une évaluation dans le contexte de maladies qui ne requièrent souvent, au départ, qu'un traitement par sulfonylurées (ou sulfamides hypoglycémiants) en monothérapie.

\section{Progéniteurs facultatifs dans le pancréas adulte}

Le pancréas est étudié depuis longtemps pour sa capacité à héberger des cellules souches ou des progéniteurs facultatifs [28], c'est-à-dire dont la capacité à proliférer et à reconstituer l'un des compartiments cellulaires du pancréas (endocrine ou exocrine) dépend de stimulus extérieurs (comme une chirurgie d'exérèse, une inflammation, des facteurs de croissance). L'enjeu est d'identifier un réservoir de cellules exploitables pour des stratégies de différenciation en cellules $\beta$, soit localement, soit après manipulation in vitro [29]. Étant donnée l'importance d'obtenir des quantités cliniquement significatives de cellules, la priorité est 


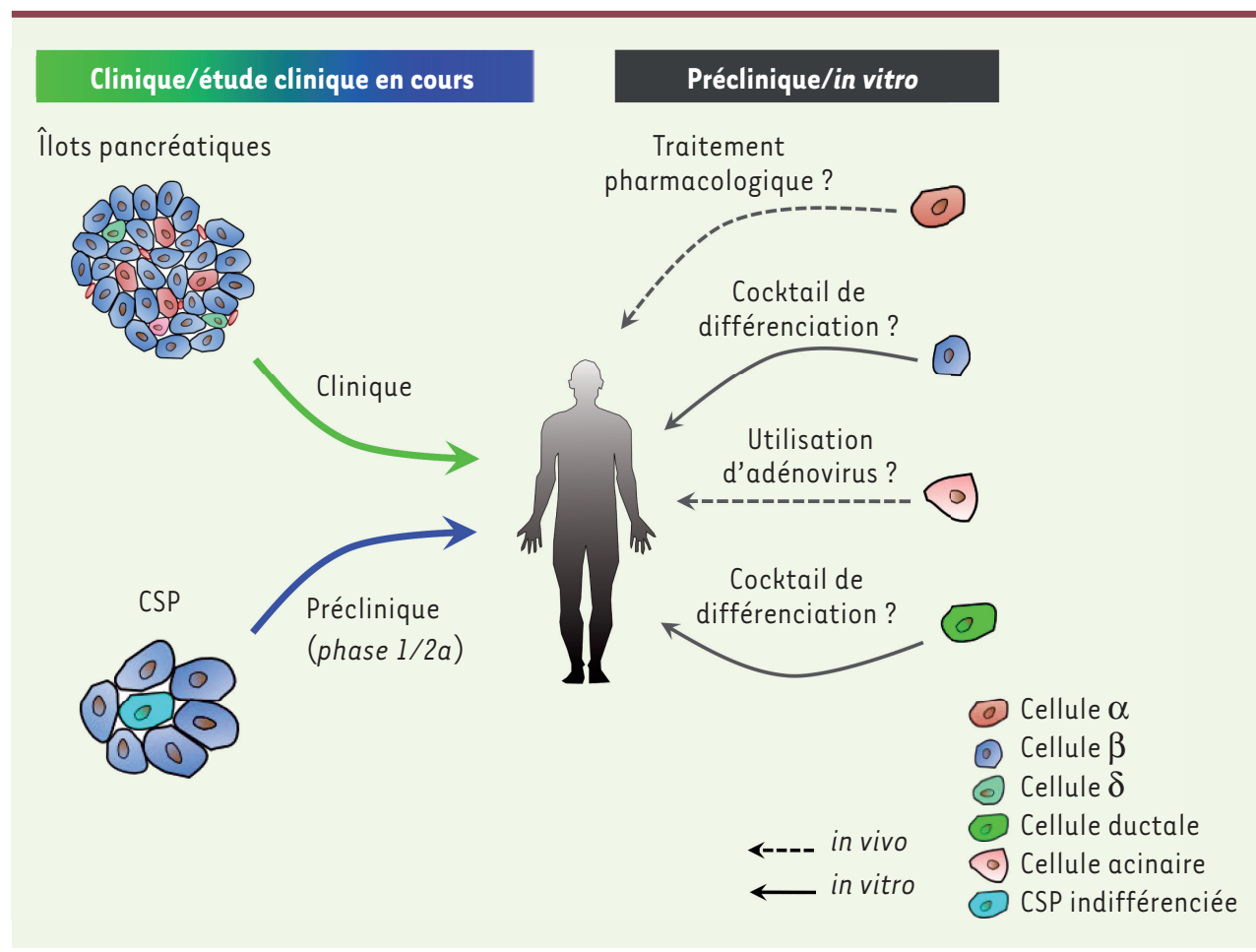

Figure 1. Sources tissulaires disponibles pour la thérapie cellulaire du diabète et méthodes envisageables ou documentées de transdifférenciation ou redifférenciation (in vivo ou in vitro) de cellules pancréatiques endocrines (cellules $\alpha$ et $\beta$ ) ou exocrines (cellules acinaires et ductales). (SP: cellules souches pluripotentes.

teurs pancréatiques (baptisés PMP pour pancreatic multipotent progenitors) expriment l'insuline mais pas le transporteur de glucose GLUT2. Cette caractéristique permet de les sélectionner au sein d'îlots par un traitement à la streptozotocine qui enrichit les donnée à l'utilisation des cellules majoritairement représentées dans le pancréas adulte.

\section{Cellules $\beta$}

Comme toutes les cellules épithéliales, les cellules $\beta$ ont des capacités de prolifération limitées. Leurs caractéristiques phénotypiques s'altèrent après la perte de leurs connexions intercellulaires (lorsqu'elles sont au sein des îlots) et leur adhérence aux surfaces de culture cellulaire. Leur réplication représente un intérêt clinique majeur, mais les stratégies pharmacologiques développées pour les stimuler pêchent par un manque de spécificité [30].

La réplication des cellules $\beta$ est néanmoins possible en forçant une transition vers un phénotype mésenchymateux, plus archaïque, un phénomène appelé transition épithélio-mésenchymateuse (TEM). La TEM des cellules $\beta$ a été démontrée en 2000 [31]. Elle a été exploitée quasi exclusivement par l'équipe de Shimon Efrat (Université de Tel Aviv, Israël) afin de stimuler une prolifération cellulaire importante tout en maintenant une faculté de différenciation in vitro et un retour possible vers un phénotype de type $\beta$ (transition mésenchymateuseépithéliale, TME) avec expression d'insuline [32]. Ces cellules TEM-TME ont des facultés de sécrétion d'insuline in vivo. L'équipe de Shimon Efrat a en effet rapporté le contrôle du diabète de souris, six semaines après transplantation de cellules ayant été traitées par un inhibiteur de la voie NOTCH [33]. Ce système, qui permet à la masse de «cellules $\beta$ » de se voir augmentée de 30 fois, nécessite néanmoins la validation par les travaux d'autres équipes.

Les îlots pancréatiques humains semblent contenir des progéniteurs qui ont la particularité de proliférer sous forme de sphères lorsqu'ils sont placés dans des conditions de culture clonale [34]. Ces progénicultures en populations de cellules insuline ${ }^{+} /$GLUT $^{\text {faible }}$ [35]. En culture, les PMP ont des facultés spontanées de différenciation en cellules $\beta$ fonctionnelles et en lignées neuronales. Ils semblent pouvoir stabiliser les glycémies de souris NOD/SCID (non-obese diabetic) severe combined immunodeficiency $)^{5}$ diabétiques sous la limite des $300 \mathrm{mg} / \mathrm{dL}^{6}$ immédiatement après transplantation [34]. La capacité des PMP à produire des lots de cellules utilisables en transplantation clinique, de même que leur efficacité à rétablir l'équilibre glycémique in vivo, restent cependant à démontrer.

\section{Cellules $\alpha$}

$\varepsilon$ ndehors des cellules $\beta$ productrices d'insuline, les îlots de Langerhans sont constitués de cellules endocrines dont la fonction est soit identifiée, comme les cellules $\alpha$ (productrices de glucagon, une hormone hyperglycémiante) et les cellules $\delta$ (qui sécrètent la somatostatine qui inhibe la production des autres hormones), soit encore largement énigmatique, comme celle des cellules PP qui sécrètent le polypeptide pancréatique [36]. Ces cellules endocrines participent de manière interactive, via un réseau neurovasculaire et des fonctions paracrines, à l'influence des îlots sur la régulation de l'homéostasie glucidique. Ces cellules endocrines hyperspécialisées possèdent ontologiquement des pro-

${ }^{5}$ Ces souris représentent un modèle de diabète de type 1.

${ }^{6} 300$ milligrammes par décilitre représentent la limite supérieure de la glycémie. 
géniteurs communs (qui expriment la neurogénine 3 [NGN3]) et sont capables à l'état mature, de plasticité. Ce phénomène inattendu a été dévoilé par l'équipe de Patrick Collombat grâce à un modèle transgénique de souris surexprimant le facteur de transcription PAX4 (paired box 4), marqueur de cellules $\beta$, dans des cellules $\alpha$ productrices de glucagon $[37,61](\rightarrow)$.

\section{$\rightarrow$ Voir la Nouvelle de F. Thorel et P.L. Herrera, $m / s$ $n^{\circ} 11$, novembre 2010, page 906} Une transition des cellules $\alpha$ - conditionnées par PAX4 - vers un phénotype $\beta$ a en effet été observée, permettant un réapprovisionnement en cellules insulino-sécrétantes suffisant pour assurer une normalisation de la glycémie de souris traitées par la streptozotocine (qui détruit les cellules $\beta$ ). Le groupe d'Herrera a, par la suite, étendu ces résultats en rapportant la capacité spontanée des cellules $\alpha$ à être activées, et converties, en cellules $\beta$ lorsque la masse des cellules $\beta$ était détruite à $99 \%$ par expression de toxine diphtérique [38]. Ils ont ainsi confirmé l'origine « $\alpha$ » des nouvelles cellules $\beta$ en montrant l'absence de ces dernières lorsque la toxine était exprimée dans les deux compartiments, $\beta$ et $\alpha$.

Étant donné qu'il est difficile d'évaluer l'existence et/ou la portée de la reprogrammation $\alpha$-vers $-\beta$ spontanée dans un contexte clinique [39], la possibilité de transposer ce mécanisme dans un contexte de thérapie cellulaire a été évaluée. Le groupe de Collombat a choisi une approche inhibitrice pour confirmer le modèle de plasticité $\alpha-\beta$. Leur étude, publiée en 2013 , démontre que l'inactivation de la spécificité des cellules $\alpha$ (qui est sous la dépendance du facteur de transcription ARX [aristaless related homeobox]) entraîne une transdifférenciation $\alpha$ vers $\beta$, en masse, qui s'étend jusqu'à un effet thérapeutique dans un modèle de diabète murin [40]. L'effet de masse obtenu provient du manque de cellules $\alpha$ (qui perdent leur identité par l'inhibition de ARX) stimulant ainsi l'activation de progéniteurs $\mathrm{NGN}^{+}$, situés en bordure des canaux pancréatiques qui empruntent le processus de spécification en lignées $\beta$. Les stratégies d'inhibition ont l'avantage de permettre d'envisager un traitement pharmacologique afin de stimuler la reprogrammation $\alpha$-vers- $\beta$, que ce soit in vivo ou en culture, bien qu'à ce stade, il n'y ait pas de modèle convaincant de manipulation ex vivo de cellules $\alpha$ humaines.

\section{Cellules acinaires}

Les cellules acinaires, qui produisent le suc digestif du pancréas, sont largement majoritaires dans cet organe. Leur potentiel de transdifférenciation en cellules $\beta$ est donc examiné depuis plusieurs décennies [41]. Les limites principales des cellules acinaires humaines sont leur fragilité et leur tendance à la dédifférenciation ex vivo, les rendant impropres à la prolifération. Ces limites ont réorienté les investigations vers des stratégies de différenciation in situ, sans recours à l'isolement et à la culture cellulaires.

Au-delà de la controverse sur les capacités des cellules acinaires à produire spontanément de nouvelles cellules $\beta$ lors de pancréatectomies ou autres lésions pancréatiques [42], il est admis que ces cellules sont capables de régénérer le pancréas exocrine [43]. Elles sont considérées à ce titre comme des progéniteurs facultatifs. Cet aspect a mené l'équipe de Douglas Melton à développer une stratégie de traitement in vivo. La possibilité d'induire une transdifférenciation acinaire-vers- $\beta$ a été examinée en forçant l'expression de facteurs de transcription gouvernant le développement embryonnaire des cellules $\beta$, à savoir PDXI (pancreatic and duodenal homeobox 1), NGN3 (neurogenin3) et MafA [44]. Lorsque ces facteurs sont transfectés, à l'aide d'adénovirus, chez des souris immunodéprimées $\left(\mathrm{Ragl}^{-/-}\right)$rendues diabétiques par la streptozotocine, de nouvelles cellules $\beta$ apparaissent rapidement dans le pancréas, au point de normaliser complètement la glycémie des animaux malades en une semaine. Malgré l'aspect transitoire de l'expression des trois facteurs par le vecteur viral, il semble que ce processus de transdifférenciation soit robuste étant donné qu'il peut être établi à long terme (jusqu'à 13 mois après le traitement [45]). Bien que révolutionnaire, et adaptée pour des cultures de tissu exocrine murin [46], cette technologie n'a pas encore fait l'objet de confirmation par d'autres groupes ou dans d'autres modèles in vivo.

Le potentiel de la transdifférenciation acinaire-vers- $\beta$ n'en est pas négligé pour autant, comme le montrent les travaux de l'équipe de Heimberg. Actifs depuis longtemps dans les investigations visant à stimuler la conversion acinaire- $\beta$ de manière pharmacologique, l'équipe avait montré l'utilité de facteurs de croissance (epidermal growth factor [EGF]) ou d'hormones (gastrine) dans la régénération des cellules $\beta$ [47]. L'extension de ces recherches a permis d'identifier deux facteurs, l'EGF et le ciliary neurotrophic factor (CNTF) qui permettent d'induire une transdifférenciation acinaire-vers- $\beta$ massive chez la plupart (65\%) des souris traitées [48]. À l'instar du modèle adénoviral que nous avons rapporté, le cocktail EGF/CNTF a permis la normalisation des glycémies des souris diabétiques (diabète induit par alloxan) seulement cinq jours après traitement par pompes osmotiques. Cette étude représente un pas supplémentaire vers la découverte de stratégies de régénération des cellules $\beta$ qui sont envisageables en clinique, du moins lorsque les effets indésirables d'un traitement systémique seront soupesés (par exemple le rôle que peut présenter l'EGF dans le cancer pancréatique [49]).

\section{Cellules ductales}

Les cellules ductales ou canalaires du pancréas sont responsables, chez l'embryon, de la formation des trois compartiments cellulaires (endocrine, acinaire et ductal) selon un principe morphogénétique d'arborescence [50]. Chez l'adulte, les cellules ductales sont quiescentes mais elles semblent contenir des progéniteurs facultatifs qui, via réactivation de l'expression 
de NGN3, pourraient participer à divers processus de régénération $[40,51]$. Le rôle et l'identité de ces progéniteurs restent imprécis et controversés [52]. Les cellules ductales ne représentent pas un modèle idéal pour des stratégies d'expansion ou de transdifférenciation in vivo, étant impliquées dans le cancer pancréatique [53].

Malgré tout, les cellules ductales possèdent plusieurs avantages majeurs par rapport aux autres types cellulaires du pancréas: elles résistent aux méthodes d'isolement tissulaire (notamment elles sont résistantes à la trypsine qui est utilisée pour séparer et isoler les cellules), à la mise en suspension, à la culture cellulaire et à la cryopréservation; elles expriment des protéines de surface qui permettent leur tri par cytométrie en flux (comme CA19-9 [cancer antigen 19-9] [54] et CD133 [55]) ; elles sont potentiellement aptes à se différencier in vitro en cellules $\beta$ comme cela a été démontré à partir de tissus humains [56]. Les cellules ductales sont donc robustes mais elles prolifèrent peu et perdent leurs caractéristiques phénotypiques après dissociation tissulaire [57]. En partenariat avec l'équipe de Susan Bonner-Weir, nous avons développé un sys-

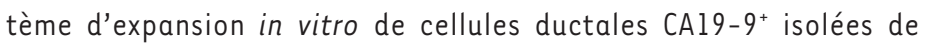
pancréas humains. Après isolement, les cellules sont incubées avec un milieu de croissance endothéliale qui a la particularité d'induire une TEM des cellules CA19-9+ qui entrent en phase de prolifération et réalisent jusqu'à 22 doublements de population [58]. Après expansion, environ 3 à $5 \%$ de ces cellules dérivées des canaux humains (en anglais human duct-derived cells ou HDDC) acquièrent des capacités de sécrétion d'insuline après 14 jours de contacts successifs avec des molécules et facteurs de croissance, selon un schéma reproduisant l'ontogenèse des cellules $\beta$. Les HDDC ont l'avantage de leur nombre (il est possible d'en collecter $10^{11}$ en 1 mois de culture) et de la résistance des cellules différenciées au stockage à $-196{ }^{\circ} \mathrm{C}$. L'objectif est actuellement d'optimiser ce potentiel de différenciation et d'en apprécier l'efficacité dans un modèle murin de diabète.

\section{Conclusion}

Le concept de la thérapie cellulaire a été établi grâce aux succès de la transplantation d'îlots, qui reste à ce jour le seul traitement ayant permis un arrêt complet de l'insuline chez des patients diabétiques de type 1. L'efficacité de ce traitement est en constante évolution mais souffre actuellement du manque de donneurs disponibles et éligibles pour la greffe, et du recours à une immunosuppression à long terme associée aux effets indésirables inacceptables dans certains contextes (notamment pédiatrique).

À ce jour, l'alternative la plus prometteuse aux îlots correspond aux CSP que quelques équipes ont réussi à porter à des niveaux de sophistication élevée en termes de fonctionnalité $\beta$, avec effet curatif dans des modèles murins de diabète. La plasticité des cellules souches a également un coût en ce qu'elle occasionne la croissance et la différenciation incontrôlée de toute cellule transplantée dans un état indifférencié. L'avènement des techniques de macro-encapsulation permettra peut-être de pallier les risques pour le patient, ce que des investigations cliniques évaluent actuellement.
Enfin, le pancréas adulte contient des cellules épithéliales qui, de par leurs propriétés de prolifération en culture ou de plasticité in vivo, pourraient constituer d'excellents candidats pour des protocoles translationnels de transdifférenciation in vitro puis transplantation ou de traitement pharmacologique, idéalement en utilisant des molécules dont l'usage est approuvé en clinique. $\diamond$

\section{SUMMARY}

Cellular therapy of diabetes: focus on the latest developments

Islet transplantation has set the ground for diabetes cell therapy and is still undergoing various developments that might improve clinical outcomes. Alternative sources for $\beta$-cell replacement strategies are now led by human pluripotent stem cells that demonstrate nearnormal $\beta$-cell features after in vitro differentiation and which can reverse diabetes in mice. Yet, their propensity for tumor formation is still unresolved. The adult pancreas is suggested as a reservoir of facultative progenitors that could represent adequate candidates for $\beta$-cell engineering, either in vivo through pharmacological treatment or after expansion in culture. This review focuses on the latest developments in protocols aiming at de novo production of functional $\beta$ cells. $\diamond$

\section{LIENS D'INTÉRÊT}

L'auteur déclare avoir bénéficié de financements de la Belgian Society for Pediatric Endocrinology and Diabetology (BESPEED), l'Institut de Recherche Clinique et Expérimentale (IREC), le Fonds National de la Recherche Scientifique (FNRS) et l'International Society for Pediatric and Adolescent Diabetes (ISPAD).

\section{RÉFÉRENCES}

1. Cameron FJ, Wherrett DK. Care of diabetes in children and adolescents: controversies, changes, and consensus. Lancet 2015 ; 385 : 2096-106.

2. Kelly WD, Lillehei RC, Merkel FK, et al. Allotransplantation of the pancreas and duodenum along with the kidney in diabetic nephropathy. Surgery 1967 ; $61: 827-37$.

3. Kaufman D. State of the art of solid organ pancreas transplantation. In : $75^{\text {th }}$ Scientific sessions of the American diabetes association. Boston, USA, 2015.

4. Redfield RR, Scalea JR, Odorico JS. Simultaneous pancreas and kidney transplantation: current trends and future directions. Curr Opin Organ Transplant $2015 ; 20: 94-102$.

5. Shapiro AM, Lakey JR, Ryan EA, et al. Islet transplantation in seven patients with type 1 diabetes mellitus using a glucocorticoid-free immunosuppressive regimen. $N$ EnglJ Med $2000 ; 343: 230-8$.

6. Barton FB, Rickels MR, Alejandro R, et al. Improvement in outcomes of clinical islet transplantation: 1999-2010. Diabetes Care 2012 ; 35 : 1436-45.

7. Balamurugan AN, Naziruddin B, Lockridge A, et al. Islet product characteristics and factors related to successful human islet transplantation from the Collaborative Islet Transplant Registry (CITR) 1999-2010. Am J Transplant 2014; 14 : 2595-606.

8. Brennan DC, Kopetskie HA, Sayre PH, et al. Long-term follow-up of the Edmonton protocol of islet transplantation in the United States. Am J Transplant 2015.

9. Ricordi C. Clinical islet transplantation update. In : $75^{\text {th }}$ Scientific sessions of the American diabetes association. Boston, USA, 2015. 


\section{RÉFÉRENCES}

10. Inverardi L. Improved graft survival in islet transplant recipients treated with G-CSF (filgrastim) and exenatide. In : $75^{\text {th }}$ Scientific sessions of the American diabetes association. Boston, USA, 2015.

11. Pepper AR, Gala-Lopez B, Pawlick R, et al. A prevascularized subcutaneous device-less site for islet and cellular transplantation. Nat Biotechnol $2015 ; 33: 518-23$.

12. Hajizadeh-Saffar $\varepsilon$, Tahamtani $Y$, Aghdami N, et al. Inducible VEGF expression by human embryonic stem cell-derived mesenchymal stromal cells reduces the minimal islet mass required to reverse diabetes. Sci Rep $2015 ; 5: 9322$.

13. Tomei AA, Villa C, Ricordi C. Development of an encapsulated stem cell-based therapy for diabetes. Exp Opin Biol Ther 2015 ; 15 : 1321-36.

14. Kroon $\varepsilon$, Martinson LA, Kadoya K, et al. Pancreatic endoderm derived from human embryonic stem cells generates glucose-responsive insulin-secreting cells in vivo. Nat Biotechnol $2008 ; 26: 443-$ 52.

15. D’Amour KA, Bang AG, Eliazer S, et al. Production of pancreatic hormone-expressing endocrine cells from human embryonic stem cells. Nat Biotechnol $2006 ; 24: 1392-401$.

16. Fujikawa T, Oh SH, Pi L, et al. Teratoma formation leads to failure of treatment for type I diabetes using embryonic stem cell-derived insulin-producing cells. Am J Pathol 2005 ; 166 : 1781-91.

17. Blum B, Benvenisty N. The tumorigenicity of diploid and aneuploid human pluripotent stem cells. Cell Cycle $2009 ; 8: 3822-30$.

18. Rezania A, Bruin JE, Riedel MJ, et al. Maturation of human embryonic stem cell-derived pancreatic progenitors into functional islets capable of treating pre-existing diabetes in mice. Diabetes $2012 ; 61: 2016-29$.

19. Bruin JE, Rezania A, Xu J, et al. Maturation and function of human embryonic stem cellderived pancreatic progenitors in macroencapsulation devices following transplant into mice. Diabetologia $2013 ; 56: 1987-98$.

20. Rezania A, Bruin JE, Arora P, et al. Reversal of diabetes with insulin-producing cells derived in vitro from human pluripotent stem cells. Nat Biotechnol $2014 ; 32$ : 1121-33.

21. Pagliuca FW, Millman JR, Gurtler M, et al. Generation of functional human pancreatic beta cells in vitro. Cell $2014 ; 159: 428-39$.

22. Schwartz SD, Regillo CD, Lam BL, et al. Human embryonic stem cell-derived retinal pigment epithelium in patients with age-related macular degeneration and Stargardt's macular dystrophy: follow-up of two open-label phase $1 / 2$ studies. Lancet $2015 ; 385: 509-16$.

23. Osafune K, Caron L, Borowiak M, et al. Marked differences in differentiation propensity among human embryonic stem cell lines. Nat Biotechnol 2008 ; 26 : 313-5

24. Mfopou JK, Chen B, Mateizel I, et al. Noggin, retinoids, and fibroblast growth factor regulate hepatic or pancreatic fate of human embryonic stem cells. Gastroenterology $2010 ; 138: 2233-45$, 45 el-14.

25. Gore A, Li Z, Fung HL, et al. Somatic coding mutations in human induced pluripotent stem cells. Nature $2011 ; 471: 63-7$.

26. Maehr R, Chen S, Snitow M, et al. Generation of pluripotent stem cells from patients with type 1 diabetes. Proc Natl Acad Sci USA 2009 ; 106 : 15768-73.

27. Teo AK, Windmueller $R$, Johansson $B B$, et al. Derivation of human induced pluripotent stem cells from patients with maturity onset diabetes of the young. J Biol Chem $2013 ; 288$ : 5353-6.

28. Jiang FX, Morahan G. Pancreatic stem cells remain unresolved. Stem Cells Dev $2014 ; 23: 2803-12$

29. Lysy PA, Weir GC, Bonner-Weir S. Making beta cells from adult cells within the pancreas. Curr Diab Rep $2013 ; 13: 695-703$.

30. Vetere A, Choudhary A, Burns SM, Wagner BK. Targeting the pancreatic beta-cell to treat diabetes. Nat Rev Drug Discov $2014 ; 13: 278-89$.

31. Gershengorn MC, Hardikar AA, Wei C, et al. Epithelial-to-mesenchymal transition generates proliferative human islet precursor cells. Science $2004 ; 306: 2261-4$

32. Russ HA, Sintov $\varepsilon$, Anker-Kitai L, et al. Insulin-producing cells generated from dedifferentiated human pancreatic beta cells expanded in vitro. PLoS One $2011 ; 6$ : e25566.

33. Bar Y, Russ HA, Sintov $\varepsilon$, et al. Redifferentiation of expanded human pancreatic beta-cell-derived cells by inhibition of the NOTCH pathway. J Biol Chem $2012 ; 287: 17269-80$.

34. Smukler SR, Arntfield ME, Razavi R, et al. The adult mouse and human pancreas contain rare multipotent stem cells that express insulin. Cell Stem Cell 2011; $8: 281-93$.

35. Razavi R, Najafabadi HS, Abdullah S, et al. Diabetes enhances the proliferation of adult pancreatic multipotent progenitor cells and biases their differentiation to more beta-cell production. Diabetes $2015 ; 64: 1311-23$.

36. Brereton MF, Vergari $\varepsilon$, Zhang $Q$, Clark A. Alpha-, delta- and PP-cells: Are they the architectural cornerstones of islet structure and co-ordination? J Histochem Cytochem $2015 ; 63: 575-91$.
37. Collombat $P, X u X$, Ravassard $P$, et al. The ectopic expression of Pax4 in the mouse pancreas converts progenitor cells into alpha and subsequently beta cells. Cell $2009 ; 138: 449-62$.

38. Thorel F, Nepote V, Avril I, et al. Conversion of adult pancreatic alpha-cells to beta-cells after extreme beta-cell loss. Nature $2010 ; 464: 1149-54$

39. Habener JF, Stanojevic V. Alpha cells come of age. Trends Endocrinol Metab $2013 ; 24: 153-63$

40. Courtney M, Gjernes $\varepsilon$, Druelle N, et al. The inactivation of Arx in pancreatic alpha-cells triggers their neogenesis and conversion into functional betalike cells. PLoS Genet $2013 ; 9$ : el003934.

41. Baeyens $L$, Bouwens $L$. Can beta-cells be derived from exocrine pancreas ? Diabetes Obes Metab 2008; 10 (suppl 4) : 170-8.

42. Desai BM, Oliver-Krasinski J, De Leon DD, et al. Preexisting pancreatic acinar cells contribute to acinar cell, but not islet beta cell, regeneration. J Clin Invest 2007 ; 117 : 971-7.

43. Murtaugh LC, Keefe MD. Regeneration and repair of the exocrine pancreas. Annu Rev Physiol $2015 ; 77$ : 229-49.

44. Zhou $Q$, Brown J, Kanarek A, et al. In vivo reprogramming of adult pancreatic exocrine cells to beta-cells. Nature $2008 ; 455: 627-32$.

45. Li W, Cavelti-Weder C, Zhang Y, et al. Long-term persistence and development of induced pancreatic beta cells generated by lineage conversion of acinar cells. Nat Biotechnol $2014 ; 32$ : 1223-30.

46. Yamada T, Cavelti-Weder C, Caballero F, et al. Reprogramming mouse cells with a pancreatic duct phenotype to insulin-producing beta-like cells. Endocrinology 2015 ; 156 : 2029-38.

47. Rooman I, Bouwens L. Combined gastrin and epidermal growth factor treatment induces islet regeneration and restores normoglycaemia in C57BI6/J mice treated with alloxan. Diabetologia $2004 ; 47: 259-65$.

48. Baeyens L, Lemper M, Leuckx G, et al. Transient cytokine treatment induces acinar cell reprogramming and regenerates functional beta cell mass in diabetic mice. Nat Biotechnol 2014 ; 32 : 76-83.

49. Ray KC, Moss ME, Franklin JL, et al. Heparin-binding epidermal growth factor-like growth factor eliminates constraints on activated Kras to promote rapid onset of pancreatic neoplasia. Oncogene $2014 ; 33: 823-31$.

50. Pan FC, Wright C. Pancreas organogenesis: from bud to plexus to gland. Dev Dyn $2011 ; 240: 530-65$.

51. Xu X, D'Hoker J, Stange G, et al. Beta cells can be generated from endogenous progenitors in injured adult mouse pancreas. Cell $2008 ; 132$ : 197-207.

52. Bonner-Weir S, Guo L, Li WC, et al. Islet neogenesis: a possible pathway for beta-cell replenishment. Rev Diabet Stud 2012 ; 9 : 407-16.

53. Esposito I, Segler A, Steiger K, Kloppel G. Pathology, genetics and precursors of human and experimental pancreatic neoplasms: an update. Pancreatology $2015 ; 15: 598-610$.

54. Yatoh S, Dodge R, Akashi T, et al. Differentiation of affinity-purified human pancreatic duct cells to beta-cells. Diabetes $2007 ; 56: 1802-9$

55. Lee J, Sugiyama T, Liu Y, et al. Expansion and conversion of human pancreatic ductal cells into insulin-secreting endocrine cells. Elife $2013 ; 2$ : e00940.

56. Bonner-Weir S, Taneja M, Weir GC, et al. In vitro cultivation of human islets from expanded ductal tissue. Proc Natl Acad Sci USA 2000 ; 97 : 7999-8004.

57. Seeberger KL, Dufour JM, Shapiro AM, et al. Expansion of mesenchymal stem cells from human pancreatic ductal epithelium. Lab Invest $2006 ; 86: 141-53$.

58. Corritore $\varepsilon$, Dugnani $\varepsilon$, Pasquale V, et al. beta-Cell differentiation of human pancreatic duct-derived cells after in vitro expansion. Cell Reprogram 2014 ; $16: 456-66$.

59. Franc C. Le diabète. Med Sci (Paris) 2013 ; $29: 711-4$.

60. Velho G, Bellanné-Chantelot C, Timsit J. Le MODY : modèle d'étude d'interactions génotype/phénotype dans le diabète de type 2. Med SCi (Paris) $2003 ; 19: 854-9$.

61. Thorel F, Herrera PL. Génération de cellules $\beta$-pancréatiques par conversion spontanée de cellules $\alpha$ chez des souris diabétiques. Med Sci (Paris) 2010 ; $26: 906-9$

\section{TIRÉS À PART}

P.A. Lysy

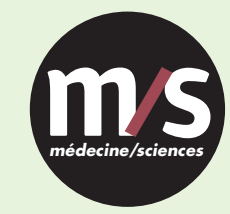

Tarifs d'abonnement $\mathrm{m} / \mathrm{s}-2016$

Abonnez-vous

à médecine/sciences
$>$ Grâce à $m / s$, vivez en direct les progrès des sciences biologiques et médicales

Bulletin d'abonnement page 422 dans ce numéro de $\mathrm{m} / \mathrm{s}$

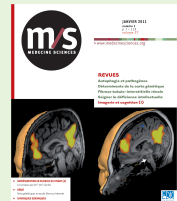

